\title{
A Failure to Reproduce the Intermediate Effect in Clinical Case Recall
}

\author{
Margaretha W. J. van de Wiel, PhD, Henk G. Schmidt, PhD, and Henny P. A. Boshuizen, PhD
}

\begin{tabular}{|c|c|}
\hline \multicolumn{2}{|c|}{ A B S TRACT } \\
\hline $\begin{array}{l}\text { Purpose. To investigate the differences between experts, } \\
\text { intermediates, and novices in diagnosing and represent- } \\
\text { ing clinical cases under various time constraints. } \\
\text { Method. Second-, fourth-, and sixth-year medical stu- } \\
\text { dents, and internists studied, diagnosed, and recalled four } \\
\text { clinical cases from internal medicine. Participants were } \\
\text { allowed to study each case for either } 3 \text { minutes, } 1 \text { minute } \\
15 \text { seconds, or } 30 \text { seconds. The study replicated in most } \\
\text { ways the } 1993 \text { clinical case recall study of Schmidt and } \\
\text { Boshuizen. } \\
\text { Results. As expected, diagnostic accuracy increased with } \\
\text { level of expertise. However, this study, failed to disclose } \\
\text { the intermediate effect in clinical case recall that was } \\
\text { found in the original study. Instead, a positive linear rela- }\end{array}$ & $\begin{array}{l}\text { tion between expertise level and case recall was found. } \\
\text { The discrepancy resulted from more elaborate recall by } \\
\text { experts in the present study. Constraining processing time } \\
\text { did not affect diagnostic accuracy, but equally affected the } \\
\text { recall performances of the participants of all expertise } \\
\text { levels. This contrasts with the earlier finding that experts' } \\
\text { recall is independent of processing time. } \\
\text { Conclusion. Although it is unclear why the experts' case } \\
\text { processing was more elaborate in the present study than } \\
\text { in the earlier study, it must be concluded that expert } \\
\text { medical knowledge is so flexibly organized that experts } \\
\text { can easily represent clinical cases in either the encapsu- } \\
\text { lated or the elaborated mode. } \\
\text { Acad. Med. 1998;73:894-900. }\end{array}$ \\
\hline
\end{tabular}

Expert performance, according to the past few decades' research, is primarily a reflection of highly specialized domain knowledge. An expert solves a problem by taking in information specific to that problem, rapidly assessing relevant knowledge stored in long-term memory, and then forming a coherent representation of the problem. 'This representation is the key to the solution of the problem.

Because experts have much more domain knowledge than do less experienced persons, it is supposed that they

Parts of this report were presented at the annual meeting of the American Educational Research Association, Atlanta, Georgia, April 12-17, 1993.

Dr. van de Wiel is postdoctoral researcher, Skillslab, Faculty of Medicine; Dr. Schmidt is professor of psychology and health professions education, Department of Psychology; Dr. Boshuizen is associate professor, Department of Educational Development and Research; all at Maastricht University, Maastricht, The Netherlands.

Correspondence and requests for reprints should be addressed to Dr. van de Wiel, Skillslab, Maastricht University, P.O. Box 616, $6200 \mathrm{MD}$ Maastricht, The Netherlands; e-mail: <m.vandewiel@educ .unimaas.nls. will form more elaborate and more complete representations of problems. To gauge the elaborateness of these representations, researchers have frequently measured problem solvers' abilities to freely recall information given in a problem. The rationale behind the use of this measure is that information encoded while solving the problem will be retrieved when recalling the problem after it is solved. Thus, it is assumed that the more elaborate the representation of the problem is, the more elaborate the recall will be. Studies of expertise in a great variety of domains, such as chess, ${ }^{2,3}$ baseball, ${ }^{4}$ bridge, ${ }^{5}$ electronic engineering, ${ }^{6}$ and computer programming, ${ }^{7}$ have indeed shown that experts remember more information from representative tasks in their domains of expertise than do less experienced participants.

Studies of expertise in the domain of medicine, however, have generally not found that recall performance increases with level of expertise. A large number of these studies, in which participants with different levels of medical training and practice have been asked to study, diagnose, and then recall clinical cases, have failed to find that level of expertise affects the elaborateness of participants' recall. ${ }^{8-12}$ Of those studies that have found an effect of expertise on clinical case recall, only a few have reported a positive linear relationship between recall and level of expertise, ${ }^{13-15}$ whereas several of the studies have reported an inverted U-shaped relationship of recall to level of expertise. ${ }^{16-20}$

In an attempt to summarize the results of those recall studies in the medical domain, Boshuizen ${ }^{16}$ performed a meta-analysis on the outcomes of 11 studies conducted between 1979 and 1987. She suggested that the inverted U-shaped relationship better described the combined results than could a monotonically increasing function. Thus, in the medical domain, it was not experts but intermediate-level participants (such as medical students) who most elaborately recalled the case ma- 
terial. This phenomenon was called the intermediate effect in clinical caserepresentation studies. ${ }^{20}$

Schmidt and Boshuizen ${ }^{20,21}$ explained that phenomenon with their theory of knowledge encapsulation. According to this theory, doctors who repeatedly apply their clinical knowledge while diagnosing and treating patients eventually restructure that knowledge. Lower-level, detailed concepts are encapsulated into a smaller number of higher-level concepts. Schmidt and Boshuizen suggested that medical experts will therefore construct more condensed representations and have less elaborately detailed recall of the cases. This is in contrast to intermediate-level students, who have acquired a large base of biomedical knowledge in the course of their medical training, but who have not yet acquired a lot of clinical experience. Such students will activate detailed biomedical knowledge in their attempts to comprehend cases and their recall will therefore be more elaborately detailed.

Schmidt and Boshuizen ${ }^{20}$ further expected that experts would process cases faster than students could, simply because the experts could immediately activate a relatively small number of comprehensive, encapsulating concepts. Those researchers, therefore, predicted that a sizable reduction in the available study time would not affect experts' encapsulated processing of cases, but would impede the elaborate processing of students. They predicted that the intermediate effect in clinical case recall would disappear when processing time was sufficiently reduced. Furthermore, they predicted that the experts' case representations and, hence, their recall protocols, would contain more highlevel encapsulating concepts than would those of students.

Schmidt and Boshuizen conducted two clinical case recall studies to provide empirical support for their theoretical position. ${ }^{20}$ In the first study, they required health sciences students, second-, fourth-, and sixth-year medical students, and internists to study a case of acute bacterial endocarditis, to formulate a diagnosis, to recall the case information, and to provide a pathophysiologic explanation of the signs and symptoms in the case. Processing time was limited to either 3 minutes $30 \mathrm{sec}$ onds, 1 minute 15 seconds, or $30 \mathrm{sec}$ onds. Although diagnostic accuracy increased linearly with level of expertise independent of processing time, the relation between recall and expertise was, as predicted, different for each processing time condition. When given $3 \mathrm{~min}$ utes 30 seconds, the medical students more elaborately recalled the details of the case than did either the health sciences students or the internists, thus illustrating the intermediate effect in clinical case recall. When given 1 minute 15 seconds, the participant groups showed no significant difference in elaborateness of recall. When the participants were given the extremely short processing time of 30 seconds, their recall showed a positive linear relationship to their levels of expertise. Under all three processing-time conditions, the more experienced participants, in addition, produced more highlevel, summarizing concepts in their recall (called "summaries" by Schmidt and Boshuizen).

In the second study, Schmidt and Boshuizen looked at the effect of first priming the participants' clinical knowledge. ${ }^{20}$ They asked health sciences students, second-, fourth-, and sixth-year medical students, and internists to tell everything they knew about endocarditis for either 3 minutes 30 seconds or 30 seconds. After completing the activation task, all participants studied for 30 seconds the same case that was used in the first study, provided a diagnosis, and recalled the information in the case. $\mathrm{Di}$ agnostic accuracy showed a positive linear relationship to level of expertise independent of activation time. The recall data clearly showed an inverted U-shaped relationship to the participants' levels of expertise when they had the opportunity to activate their knowledge for 3 minutes 30 seconds. More- over, the medical students remembered less from the case when they had less time to activate their knowledge. These results suggest that the understanding of a clinical case by intermediates depends on the activation of detailed pathophysiologic knowledge, whereas experts' understanding is independent of this kind of elaborate processing.

Although those two recall studies strongly support the theory that knowledge becomes encapsulated in the development from student to expert physician, they were performed with only one clinical case, that of acute bacterial endocarditis. The same case, moreover, was used in two studies of Patel and colleagues that also demonstrated an intermediate effect in clinical case recall. ${ }^{18,19}$ In order to broaden the base of studies documenting the generalizability and robustness of the phenomenon of the intermediate effect, we felt it appropriate to replicate the 1993 study of Schmidt and Boshuizen, this time employing different case materials.

\section{METHOD}

\section{Participants}

The participants in our study were 96 students and physicians at Maastricht University in The Netherlands: $24 \mathrm{sec}$ ond-year, 24 fourth-year, and 24 sixthyear medical students, ${ }^{*}$ and 24 internists with at least four years of experience in internal medicine. We subdivided each group of 24 into three groups of eight and assigned each subgroup to one of three time-constraint conditions. The subdivision was random for the three student groups, whereas it was balanced for

\footnotetext{
*The first and second years of medical education at Maastricht University emphasize the acquisition of basic science knowledge, aiming to provide students a good understanding of physiology, anatomy, and general pathophysiology. In the third and fourth years, education centers around clinical problems, addressing clinical and patho. physiologic knowledge of disease. The fifth and sixth years consist of different clerkships in the clinic.
} 
the internists based on their subspecialties. The participants received a small compensation.

\section{Materials}

The materials consisted of four booklets, each containing a description of a clinical case and two blank response sheets. Each clinical case description reported some contextual information, the complaint, findings from taking a history and performing a physical examination, the relevant laboratory data, and some additional findings. The four clinical cases presented were pheochromocytoma, stomach carcinoma, heart failure, and liver cirrhosis. These were based on cases of actual patients and were presented following a standard patient-presentation format. Except for pheochromocytoma, these types of cases are fairly common in medical practice. The case descriptions were about half a page long and consisted of $33,42,43$, and 35 propositions, or small, meaningful information units, respectively. An example is the case of stomach carcinoma:

A 76-year-old male, who lives with his wife in a home for the elderly, is complaining of nagging pain in his upper abdomen for the last four months. His weight has decreased $9 \mathrm{~kg}$ in the last year. He is referred to a physician.

Further history taking reveals that he does not complain of nausea, but does complain of heartburn. His stool is sometimes a bit dark, and he has some difficulty swallowing. He gets tired easily. He complains of claudication, with a walking distance limited to $750 \mathrm{~m}$. He smokes 80 cigarettes a week.

Physical examination shows a healthy-looking man, weighing $52 \mathrm{~kg}$ and $1.58 \mathrm{~m}$ tall. His pulse is 84 per minute, and the blood pressure is $170 / 80 \mathrm{~mm} \mathrm{Hg}$. Auscultation reveals a loud aorta-sclerosis murmur. Over the abdominal aorta a murmur is heard, radiating to the iliac vessels. No abnormal palpable masses in the abdomen.
Laboratory results show an ESR of $41 \mathrm{~mm} / \mathrm{h}$ (normal: $<7 \mathrm{~mm} / \mathrm{h}$ ) and a hemoglobin of $8.2 \mathrm{mmol} / \mathrm{L}$ (normal: $8.5-11.0 \mathrm{mmol} / \mathrm{L}$ ). Creatinine is 84 $\mu \mathrm{mol} / \mathrm{L}$ (normal: $71-110 \mu \mathrm{mol} / \mathrm{L}$ ), ASAT (SGOT) $13 \mathrm{U} / \mathrm{L}$ (normal: $<40$ U/L), ALAT (SGPT) 7 U/L (normal: $<40$ U/L), LDH $354 \mathrm{U} / \mathrm{L}$ (normal: 200-450 U/L), alkaline phosphatase $77 \mathrm{U} / \mathrm{L}$ (normal: 30-125 U/L), gamma-GT $15 \mathrm{U} / \mathrm{L}$ (normal: $<50$ $\mathrm{U} / \mathrm{L}$ ), total protein $57 \mathrm{~g} / \mathrm{L}$ (normal: 65-97 $\mathrm{g} / \mathrm{L})$, albumin $32 \mathrm{~g} / \mathrm{L}$ (normal: $35-55 \mathrm{~g} / \mathrm{L}$ ). Ultrasonography of the abdomen shows no abnormalities.

\section{Procedure}

The participants were told to study each case carefully, to produce a diagnosis, and then to write down what they remembered from the case. We emphasized the task of diagnosis so that the participants would not view this as an exercise in memorization and recall. We first gave them an example case to familiarize them with the format and the study time allowed. Depending on the subgroups to which they were assigned; the participants were given the opportunity to study each case for either 3 minutes, 1 minute and $15 \mathrm{sec}$ onds, or 30 seconds. The participants were free to use as much time as they needed for the assignments. We staggered the order of case presentations for balance.

\section{Analysis}

We scored the participants' diagnoses on a scale of 0 (completely inaccurate diagnosis) to 6 (completely accurate diagnosis), awarding points for accurate diagnostic elements. For example, the complete diagnosis for the stomach carcinoma case was "stomach carcinoma and atherosclerosis." Four points were given if the diagnosis contained the term "stomach carcinoma" and two points were given if it contained the term "atherosclerosis." Two points were given for par- tially correct diagnoses, such as "upper digestive tract carcinoma."

Based on a technique of proposition analysis for medical protocols, ${ }^{22}$ recall protocols were segmented into small, meaningful information units, or propositions. These propositions consisted of two concepts connected by a qualifier, such as specification (spec), location (loc), or time information (temp). For instance, the protocol fragment "The heart is enlarged to all sides, and auscultation reveals a holosystolic murmur at the apex radiating towards the axilla" consists of five propositions: (1) heart-spec (enlarged to all sides); (2) auscultationspec (murmur); (3) murmur-spec (holosystolic); (4) murmur-loc (at the apex); and (5) at the apex-spec (radiating towards the axilla). We determined the participants' total recall scores by counting the numbers of propositions in the recall protocols that literally recalled, slightly imprecisely recalled, paraphrased, or summarized one or more propositions in the case materials. The numbers of summaries, that is, inferences referring to more than one proposition, were also counted separately. For example; the five propositions in the above recall fragment matched literally the original text, giving a recall score of 5 and a summary score of 0 . However, the same information might be recalled as "The heart is enlarged to all sides and auscultation reveals mitral valve insufficiency." In this latter recall fragment, the proposition "auscultation reveals mitral valve insufficiency" summarizes four propositions from the original text (auscultation reveals a holosystolic murmur at the apex radiating towards the axilla), giving a recall score of 2 and a summary score of 1.

The protocols were scored by the first author (MWJvdW) and a research assistant, whose interrater agreement exceeded .98. Data were analyzed by repeated-measures MANOVA with expertise level and processing time as between-subjects factors and cases as within-subjects factor. Polynomial con- 
trast analysis was used to test specific hypotheses.

\section{Results}

\section{Diagnostic Accuracy}

The upper panel of Figure 1 shows the participants' diagnostic accuracy as a function of their levels of expertise and processing times. A significant main effect of level of expertise on diagnostic accuracy was found, $F(3,84)=70.59$, $\mathrm{MS}_{e}=186.5, \quad p=.0001$. Polynomial contrast analy-sis revealed only a significant linear trend, $F(1,84)=211.6$, $M S_{e}=599.0, p=.0001$. Thus, the more experienced the participants, the better diagnoses they made. An increase in diagnostic accuracy is considered one of the most stable consequences of increasing medical expertise. Therefore, it can be concluded that the participants' tasks in this experimental setting reflected their expertise in internal medicine. The effect of processing time was not signifcant, $F(2,84)=.52, \mathrm{MS}_{\mathrm{e}}=1.39, p=.59$. Similar findings were reported by Schmidt and Boshuizen..$^{20}$ These findings suggest that the quality of the diagnosis is largely independent of processing time, at least within the limits of the processing times allowed in these studies. The analysis further showed a significant case effect $(F(3,252)=49.42$, $\mathrm{MS}_{e}=108.7, p=.0001$ ), and a sig. nificant interaction between cases and levels of expertise $(F(9,252)=5.03$, $M S_{e}=11.06, p=.0001$ ). For each of the four cases, polynomial contrast analysis revealed significant linear trends of diagnostic accuracy with the level of expertise. However, some of the cases seemed to be more difficult than others; participants scored lowest overall on the case of stomach carcinoma (2.0) and highest on the case of liver cirrhosis (4.6).

\section{Recall}

The relationships between the average number of propositions recalled, level of
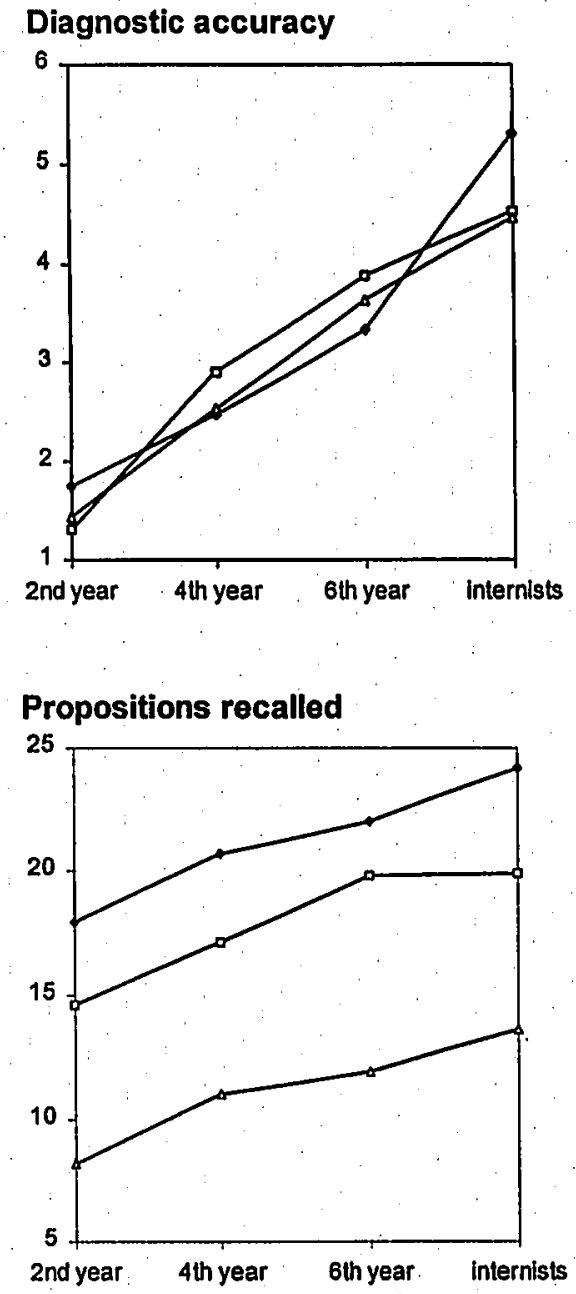

Processing time

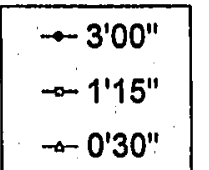

Summaries in recall

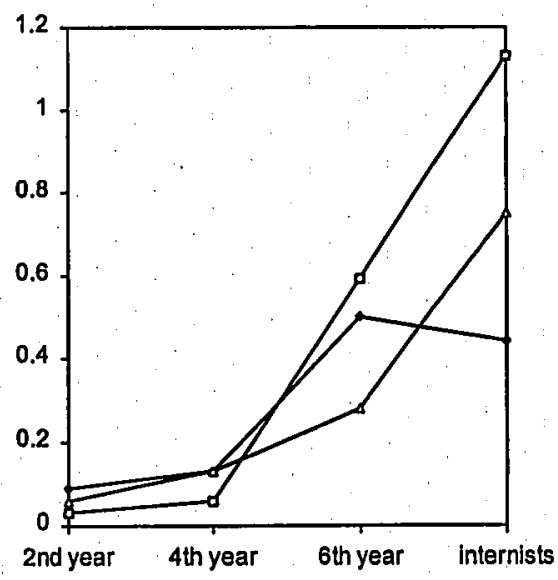

Level of expertise

Figure 1. Diagnosis and recall in four clinical cases as a function of participants' expertise levels and processing times. The upper panel shows the participants' mean diagnostic accuracy. The middle panel shows the mean number of propositions recalled. The lower panel shows the mean number of summaries produced. 
expertise, and processing time are depicted in the middle panel of Figure 1 , showing a positive, linear recall function of expertise. Analyses revealed a strong overall effect of level of expertise, $F(3,84)=10.27, M S_{e}=.39, p=$ .0001 , which could be specified as a significant linear trend, $F(1,84)=29.90$, $M S_{\mathrm{e}}=1.15, p=.0001$, without a significant quadratic component, $F(1,84)=$ $.88, \mathrm{MS}_{e}=.034, p=.35$. This means that the higher the level of expertise, the more elaborate the participant's recall was. Processing time had also a significant effect on recall, $F(2,84)=$ 59.76, $\mathrm{MS}_{e}=2.29, p=.0001$, while no interaction effect of processing time and level of expertise occurred, $F(6,84)=$ $.006, \mathrm{MS}_{\mathrm{e}}=.037, p=.99$. All participants provided more elaborate recall protocols under longer processing time conditions. These effects of expertise level and processing time on recall are even more convincing when we examine these effects for each separate case by means of ANOVA. It was found that both the main effect of expertise level and the main effect of processing time were significant for each case. In addition, no significant interaction effect occurred. For each case, the data showed a positive linear relationship of recall to level of expertise, with a significant linear component. With one exception, the quadratic component of recall with expertise level was not significant. The case of liver cirrhosis showed a significant quadratic component, $F(1,84)=8.89, M S_{e}=126.8, p=$ .0037 , which was due to a relatively low recall performance of the internists in the 1 minute 15 seconds processing time condition for this case.

\section{Summaries}

The bottom panel of Figure 1 displays the average numbers of summaries produced in the recall protocols as a function of level of expertise and processing time. The main effect of level of expertise on number of summaries produced was significant, $F(3,84)=27.88, M S_{e}=$ $10.62, p=.0001$; also present were a linear component, $\quad F(1,84)=77.44$, $M S_{e}=29.50, p=.0001$, and a quadratic component, $\quad F(1,84)=4.62, \quad M S_{e}=$ $1.76, p=.035$. In addition, pairwise comparisons between the four expertise groups by the Student-Newman-Keuls test (significance level of .05), revealed that sixth-year students produced more summaries than did second- and fourthyear students, and internists, in turn, produced more summaries than did sixth-year students. Processing time had no overall significant effect on the number of summaries produced, $F(2,84)=$ $2.75, \mathrm{MS}_{e}=1.05, \mathrm{p}=.069$.

\section{Discussion}

The Schmidt and Boshuizen study ${ }^{20}$ showed that medical experts, when given a relatively long time to process a clinical case, remembered fewer details from the case than did advanced medical students. However, the experts' recall was not affected by constraining their processing time, whereas the students' recall was. In addition, Schmidt and Boshuizen found that, although the number of detailed propositions recalled by the experts was lower than that recalled by the advanced students, the experts produced more summaries. Schmidt and Boshuizen assumed that these findings were the result of encapsulations in the knowledge bases of experienced physicians. As stated earlier, because experienced physicians tend to process case information using knowledge in encapsulated mode, their recall was expected to be short and summarized rather than reproducing the information in the case. Students, on the other hand, did not yet have knowledge available in encapsulated format and, therefore, had to process the information in an elaborate fashion.

The present experiment largely failed to replicate those findings. We found no intermediate effect. A comparison of the two data sets suggests that the dis- crepancy in recall performances between the two studies stemmed from a better recall performance by the experts in our study and not from a poorer recall performance by the intermediate students: In the present study, under the long processing time condition, the average percentages of propositions recalled by the fourth-year students, sixth-year students, and internists were $55 \%, 58 \%$, and $64 \%$, respectively, while those outcomes in the original study were $56 \%, 51 \%$, and $31 \%$, respectively. These differences suggest that the experts in the present study constructed more elaborate case representations than did the experts in the 1993 study.

The numbers of summaries produced in recall by the participants in our study compare more closely to those found in the previous study. In both studies, the experts generally produced significantly more summaries than did the students. Schmidt and Boshuizen interpreted these data as an indication that experts process cases in encapsulated mode to a larger extent than do students. We should note, however, that the average number of summaries produced in the present study $(.8)$ was considerably smaller than that in the study of Schmidt and Boshuizen (2.2).

The question, then, is: How can we explain our failure to reproduce an intermediate effect in clinical case recall? This question is particularly pertinent because the present findings are generally in agreement with those from other domains, such as chess, baseball, bridge, electronic engineering, and computerprogramming. ${ }^{2-7}$ On the other hand, intermediate effects do not represent an isolated phenomenon; a large number of clinical case recall studies employing various experimental procedures and materials have found that experts provided less elaborate recall protocols than did intermediate students. ${ }^{16,19,20}$ In addition, there is evidence from "think-aloud" studies that the granularity of the concepts used by experienced physicians while thinking about a case 
is indeed coarser than the granularity of those applied by students. ${ }^{23}$ This was also evidenced by post-hoc explanation studies that showed that experts in explaining the signs and symptoms in a clinical case applied more high-level explanatory concepts and less detailed biomedical concepts than did students. ${ }^{20,22}$ Furthermore, there is evidence from other domains that experts tend to chunk information in larger units for processing. 2,24

If we assume that the results of both studies are meaningful and the discrepancy in outcomes is not due to uncontrollable factors in the recall process itself, it is necessary to examine the differences between the two experiments in more detail.

First, the present experiment used four cases instead of one. Presentation of four cases in a sequential fashion may have given the participants time to adapt to the requirements of the experiment and to pay special attention to information they would otherwise have processed in an encapsulated mode. If this were true, one would expect recall performance to improve during the course of the experiment, later cases being better recalled than earlier ones. Analysis of the effect of presentation order on the percentage of case propositions recalled indeed showed a significant effect, $F(3,372)=2.95, \mathrm{MS}_{\mathrm{e}}=$ $.042, p=.033$. This position effect, however, cannot account for the failure to replicate the intermediate effect, because recall performance on the first case already showed a significant, positive, linear relationship with level of expertise: ANOVA of all cases that were presented first revealed an overall effect of expertise level on percentage of propositions recalled, $F(3,72)=5.52$, $M S_{e}=.075, p=.0018$, which could be specified as a linear trend, $F(1,72)=$ $15.40, \mathrm{MS}_{\mathrm{e}}=.21, \mathrm{p}=.0002$, without a quadratic component, $F(1,72)=.81$, $\mathrm{MS}_{e}=.011, p=.37$.

Second, the four clinical cases used in the present experiment differed from the case used in the experiment of Schmidt and Boshuizen. ${ }^{20}$ It might be possible that the four cases used in this experiment were more difficult than the endocarditis case used by Schmidt and Boshuizen and, therefore, required elaborate processing of the data even by expert physicians. If this were true, it might be expected that the experts needed more time to process the cases in this experiment and diagnosed them less accurately. Informal observations did show that most experts in the 3-minutes condition used all the time available to study the cases, whereas in the Schmidt and Boshuizen study the experts often stopped processing after only 1 minute 30 seconds, presumably because they did not need more time. The data on the quality of the diagnoses; however, do not suggest that the cases in the present experiment were more difficult than the endocarditis case used by Schmidt and Boshuizen: the mean diagnostic accuracies of the experts were 4.8 in the present study and only 3.1 in the study of Schmidt and Boshuizen.

\section{Conclusion}

The present study consistently produced a recall function that increased with level of expertise for four cases of internal medicine. This suggests that the experts formed more elaborate case representations than did the students. Reduction of processing time, moreover, affected not only the recall performance of the students but also that of the experts: all participants provided less elaborate recall protocols when they had less time to study the cases. This suggests that even the expert physicians processed the cases more elaborately when more study time was available. These findings contradict the earlier findings of Schmidt and Boshuizen, ${ }^{20}$ which suggested encapsulated processing of case information by experts. A comparison of the recall performances of the experts in the two studies indeed suggests that the experts in the present study processed case information more elaborately than did the experts in the study by Schmidt and Boshuizen. This was also evidenced by the longer processing times of the experts in the present study and by the lower number of summaries produced in their recall protocols. Although it remains unclear why the experts in the present study elaborately processed the cases, we must conclude that expert medical knowledge is so flexibly organized that experts can represent clinical cases both in encapsulated mode and in elaborated mode. Further research should focus on the mechanisms underlying this flexibility in knowledge organization and use.

The authors thank Prof. Dr. P. W. de Leeuw, Department of Internal Medicine, Maastricht University, for his help in constructing the clinical cases, and M. de Vries for her assistance in scoring the data.

\section{REFERENCES}

1. Van Dijk TA, Kintsch W. Strategies of Discourse Comprehension. New York: Academic Press, 1983.

2. Chase WG, Simon HA. Perception in chess, Cogn Psychol. 1973;4:55-81.

3. De Groot AD. Thought and Choice in Chess. 2nd ed. The Hague, The Netherlands: Mouton Publishers, 1978.

4. Spilich GJ, Vesonder GT, Chiesi HL, Voss JF. Text-processing of domain-related information of individuals with high and low domain knowledge. J Verb Learn Verb Behav. 1979;18:275-90.

5. Charness N. Components of skill in bridge. Can J Psychol. 1979;33:1-16.

6. Egan DE, Schwartz BJ. Chunking in recall of symbolic drawings. Mem Cogn. 1979;7:149-58.

7. McKeithen KB, Reitman JS, Rueter $\mathrm{HH}$, Hirtle SC. Knowledge organization and skill differences in computer programmers. Cogn Psychol. 1981; 13:307-25.

8. Claessen HFA, Boshuizen HPA. Recall of medical information by students and doctors. Med Educ. 1985;19:61-7.

9. Hassebrock Fi Johnson PE, Bullemer P, Fox PW, Moller $\mathrm{JH}$. When less is more: representation and selective memory in expert problem solving. Am J Psychol. 1993;106:155-89.

10. Muzzin LJ, Norman GR, Jacoby LL, Feighener 
JW, Tugwell P, Guyatt GH. Manifestations of expertise in recall of clinical protocols. Proc Annu Conf Res Med Educ. 1982:163-8.

11. Muzzin LJ, Norman GR, Feightner JW, Tugivell P. Expertise in recall of clinical protocols in two specialty areas. Proc Annu Conf Res Med Educ. 1983:122-8.

12. Norman GR, Jacoby LL, Feightner JW, Campbell EJM. Clinical experience and the structure of memory. Proc Annu Conf Res Med Educ. 1979: 214-9.

13. Coughlin LD, Patel VJ. Processing of critical information by doctors and medical students. J Med Educ. 1987;62:818-28.

14. Hobus PPM, Schmidt HG, Boshuizen HPA, Patel VL. Contextual factors in the activation of first dingnostic hypotheses: expert-novice differences. Med Educ. 1987;21:471-6.

15. Norman GR, Brooks LR, Allen SW. Recall by expert medical practitioners and novices as a record of processing attention. J Exp Psychol Learn Mem Cogn. 1989;15:1166-74.
16. Boshuizen HPA. De Ontwikkeling van Medische Expertise: Een Cognitief-Psychologische Benadering [The Development of Medical Expertise: A Cognitive Psychological Approach]. Doctoral dissertation. Haarlem, The Netherlands: Thesis publishers, 1989.

17. Patel VL, Frederiksen CH. Propositional representations of medical texts by medical students and physicians. Paper presented at the annual meeting of the American Educational Research Association, New Orleans, LA, 1984. :

18. Patel VL, Groen GJ. The general and specific nature of medical expertise: a critical look. In: Ericsson A, Smith J (eds). Toward a General Theory of Expertise: Prospects and Limits. Cambridge, U.K.: Cambridge University Press, 1991:93-125.

19. Patel VM, Medley-Mark V. Relationship between Representation of Textual Information and Underlying Problem Representation in Medicine. Cognitive Research Series, report no.
CME86-CS1. Montreal; Quebec, Canada: McGill University, Centre for Medical Education, 1986.

20. Schmidt HG, Boshuizen HPA. On the origin of intermediate effects in clinical case recall. Mem Cogn. 1993;21:338-51.

21. Schmidt HG, Boshuizen HPA. Encapsulation of biomedical knowledge. In: Evans AE, Patel VL (eds). Advanced Models of Cognition for Medical Training and Practice. New York: Springer Verlag, 1992:265-82.

22. Patel VL, Groen GJ. Knowledge based solution strategies in medical reasoning. Cogn Sci. 1986; 10:91-116.

23. Boshuizen HPA, Schmidt HG. On the role of biomedical knowledge in clinical reasoning by experts, intermediates and novices. $\operatorname{Cogn} \mathrm{Sci}$. 1992;16:153-84.

24. Koedinger $\mathrm{KR}$, Anderson JR. Abstract planning and perceptual chunks: elements of expertise in geometry. Cogn Sci. 1990;14:511 - 50 . 